\title{
Diabetes: impaired damage control
}

\author{
N. C. Schaper • B. Havekes
}

Received: 13 July 2011 / Accepted: 6 October 2011 / Published online: 12 November 2011

(C) The Author(s) 2011. This article is published with open access at Springerlink.com

\begin{abstract}
A coordinated response by the innate immune system, (micro)circulation and nervous system is needed to limit tissue destruction and to initiate reparative processes after tissue damage. Alterations in danger signals in diabetes can be an important cause of the excessive tissue loss and defective tissue repair after injury and can contribute to the higher rates of cardiac failure after myocardial infarction, more severe tissue loss in the case of peripheral ischaemia and impaired wound healing. Here we discuss the mechanisms underlying this impaired damage control in diabetes, with an emphasis on the proinflammatory cytokine high mobility group box 1 and the potential role of dipeptidyl peptidase IV inhibition in improving repair responses.
\end{abstract}

Keywords Cytokines · Diabetic complications · DPP-IV · Endothelium · Oral pharmacological agents · Pathogenetic mechanisms

N. C. Schaper $(\bowtie) \cdot$ B. Havekes

Department of Internal Medicine, Division of Endocrinology, Maastricht University Medical Centre,

PO Box 5600, 6202 AZ Maastricht, the Netherlands

e-mail: n.schaper@mumc.nl

N. C. Schaper

Care and Public Health Research Institute (CAPHRI),

Maastricht University Medical Centre,

Maastricht, the Netherlands

N. C. Schaper

Cardiovascular Research Institute Maastricht (CARIM),

Maastricht University Medical Centre,

Maastricht, the Netherlands

B. Havekes

Nutrition and Toxicology Research Institute Maastricht (NUTRIM), Maastricht University Medical Centre,

Maastricht, the Netherlands
Abbreviations
CAD Coronary artery disease
DPP-IV Dipeptidyl peptidase IV
EPC Endothelial progenitor cell
HIF-1 $\alpha$ Hypoxia-inducible factor 1
HMGB1 High mobility group box 1
RAGE Receptor for AGEs
SDF-1 Stromal cell-derived factor 1
VEGF Vascular endothelial growth factor

High mobility group box 1 (HMGB1) is a ubiquitous nuclear protein that stabilises DNA and facilitates gene transcription [1]. As one of several proteins secreted in response to cellular stress or infection, HMGB1 is also an important danger signal linking tissue necrosis, inflammation and tissue repair [1]. After tissue injury, it can be passively released during cellular necrosis by almost all cells containing nuclei. HMGB1 was originally described as a late mediator of septic shock, and both non-immune and inflammatory cells can actively secrete HMGB1 [1].

The stimuli for secretion are diverse and include exogenous pathogen-derived molecules, such as lipopolysaccharide, and cytokines [1]. Other stimuli for HMGB1 secretion are hypoxia, ischaemia-reperfusion injury and hyperglycaemiainduced cellular damage of endothelial cells $[1,2]$. Once released into the extracellular milieu, HMGB1 can bind to the receptor for AGEs (RAGE) and to members of the Tolllike family of receptors, resulting in the subsequent secretion of several cytokines, such as IL- $1 \beta$, TNF- $\alpha$ and monocyte chemoattractant protein 1. Moreover, in response to HMGB1, cell adhesion molecules are expressed on endothelial cells, triggering or augmenting inflammatory responses and increasing endothelial permeability [1]. Furthermore, HMGB1 stimulates endothelial cell migration 
and sprouting and recruits endothelial progenitor cells (EPCs) and haematopoietic stem cells to sites of tissue injury to stimulate neovascularisation [1]. The potential therapeutic value of HMGB1 in tissue repair has recently been studied in several animal models. HMGB1 enhanced angiogenesis, stimulated cardiomyogenesis and improved cardiac function after myocardial infarction in non-diabetic animals [1]. Moreover, in diabetic animals, HMGB1 administration improved wound healing after punch biopsy and increased perfusion recovery after femoral ligation [3, 4]. In this issue of Diabetologia, Marchetti and co-workers report that the activity of HMGB1 may be enhanced by treatment with dipeptidyl peptidase IV (DPP-IV) inhibitors [5], which means that DPP-IV inhibition could have beneficial effects beyond improving glycaemic control.

Marchetti and colleagues report that HMGB1 is cleaved and probably inactivated at its N-terminal region by DPP-IV [5]. In type 2 diabetic patients, DPP-IV inhibitors are widely used to prevent the inactivation of the incretin hormones, glucagonlike peptide 1 and gastric inhibitory polypeptide [6]. Although DPP-IV has many substrates in vitro, relatively few have so far been found to be physiological substrates in vivo [6]. DPP-IV is widely distributed throughout the body and exists as both a membrane-anchored cell-surface peptidase and a soluble form in plasma [6]. Marchetti et al. have now shown that DPP-IV inhibits HMGB1-induced endothelial cell migration in vitro as well as HMGB1-induced neovascularisation in vivo [5]. The authors also performed a randomised trial in which people with type 2 diabetes were treated with a DPP-IV inhibitor (sitagliptin or vildagliptin) or placebo. Their findings confirmed earlier reports that HMGB1 levels are elevated at baseline in patients with type 2 diabetes compared with healthy individuals [7], although the circulating form of the protein lacked its $\mathrm{N}$ terminal region. DPP-IV inhibitor treatment resulted in increased levels of uncleaved HMGB1 compared to placebo [5]. While the report is marred by the failure of the authors to describe either the randomisation procedure used or the blinding of investigators or participants, these results suggest that DPP-IV inhibitors can raise levels of the active form of circulating HMGB1 and could theoretically improve the outcome of cardiovascular events and wound healing in patients with diabetes. However, do we want to raise the levels of a proinflammatory signal such as HMGB1 in diabetic patients? Could increasing HMGB1 activity prove to be a double-edged sword?

Increased levels of circulating inflammatory mediators is a common feature of type 2 diabetes, together with the increased expression of RAGE and Toll-like receptors and the upregulation of inflammatory cytokine production by circulating monocytes [7]. Hyperglycaemia can activate intracellular proinflammatory pathways, such as nuclear factor $\mathrm{kB}$ in endothelial cells and monocytes [8]. These changes are probably the result of the increased production of highly reactive precursors of AGEs and increased oxidative stress. Recruitment and activation of inflammatory cells is a crucial step in atherosclerosis; in human atherosclerotic plaques HMGB1 is secreted by endothelial cells, foam cells and smooth muscle cells [9]. Serum levels of HMGB1 have been associated with coronary artery disease (CAD) in both diabetic and non-diabetic patients, with the highest levels observed in diabetic patients with CAD [10]. Neutralising HMGB1 with a monoclonal antibody reduced the size of atherosclerotic lesions in $A p o e^{-/-}$mice by $50 \%$, thus demonstrating the potentially harmful effects of raising HMGB1 levels [11]. Furthermore, HMGB1 levels are elevated in the eyes of patients with diabetic proliferative retinopathy undergoing surgery for retinal detachment [12].

It is therefore possible that any new therapy that aims to raise the levels of HMGB1 or other inflammatory mediators in order to stimulate tissue repair in one tissue might also lead to unwanted effects in other tissues. However, such effects have not been observed in any clinical study with DPP-IV inhibitors so far. On the contrary, some recent animal data suggest that DPP-IV inhibitors might have cardioprotective effects, restore tissue perfusion and promote homing of stem cells to injured areas $[13,14]$.

Given its diverse substrates, several survival and reparative pathways activated during tissue injury in addition to HMGB1 might be enhanced by DPP-IV inhibition. One attractive candidate is the hypoxia-inducible factor $1 \alpha$ (HIF-1 $\alpha)$ pathway, which is downregulated by hyperglycaemia, as recently reviewed by Bento in this journal [8]. The occlusion of large blood vessels or damage to smaller vessels in a skin wound results in cellular hypoxia with activation of HIF-1 $\alpha$. Downregulation of this adaptive response to hypoxia is probably one of the central defects in diabetes, resulting in an impaired angiogenic response to tissue ischaemia and injury [8]. Recent studies suggest that increased intracellular AGE formation and oxidative stress disrupts this HIF- $1 \alpha$ activation [8]. Although this hypothesis is mainly based on animal studies, there are some clinical data supporting this concept. Levels of HIF-1 $\alpha$ were markedly decreased in biopsies from three diabetic patients with diabetic foot ulcers compared with two normoglycaemic patients with a venous ulcer [15], and several clinical studies found impaired downstream signals of the HIF- $1 \alpha$ pathway in diabetic patients, such as nitric oxide, vascular endothelial growth factor (VEGF) and stromal cell-derived factor 1 (SDF-1) [8]. SDF-1 is a chemokine with potent angiogenic properties that stimulates bone marrow mobilisation of EPCs and recruits these cells to ischaemic tissues [8]. Recent studies suggest that SDF-1 might be an interesting therapeutic target for the treatment of ischaemic heart disease and peripheral arterial disease. Overexpression of the gene encoding SDF-1 in mice with an ischaemic limb promoted mobilisation of EPCs, 
enhanced angiogenesis and increased peripheral blood flow [15]. Local administration of SDF-1 in wounds of diabetic mice resulted in a twofold increase in the number of circulating EPCs, increased homing of these cells and improved wound healing [16]. Interestingly, SDF-1 is a substrate for DPP-IV [6], and 4 weeks of sitagliptin treatment resulted in an increase in circulating SDF-1 levels and EPCs in type 2 diabetic patients [17]. The use of DPP-IV inhibitors to improve the poor outcome of myocardial infarction and ischaemic diabetic foot ulcers in diabetes is therefore an attractive concept to explore in clinical studies.

There is one final caveat. Any therapy aiming to enhance downstream signalling of reparative pathways in patients with diabetes, e.g. by use of chemokines, growth factors or DPP-IV inhibitors, has the limitation that it is unlikely to correct the underlying cellular defects. Increased intracellular AGE formation and oxidative stress induce proinflammatory changes with disruption of the HIF- $1 \alpha$ pathway, as described above. In addition, AGEs and oxidative stress also have negative downstream consequences, such as the impaired EPC response to SDF-1 [18], the impaired proliferative response of fibroblasts to growth factors [19] and VEGF resistance of circulating monocytes [20]. This resistance to reparative signals might explain the unimpressive results of growth factor therapy in diabetic patients with poorly healing diabetic foot ulcers. Recent animal studies have shown that angiogenesis and wound healing can be restored by decreasing intracellular oxidative stress $[18,21]$. The challenge is therefore to develop new strategies that correct the intracellular metabolic defects in order to improve the poor outcome of cardiovascular disease and foot ulcers in patients with diabetes.

Acknowledgements Both authors were responsible for interpretation of data and/or literature and wrote and approved the final version for publication.

Duality of interest The authors declare that there is no duality of interest associated with this manuscript.

Open Access This article is distributed under the terms of the Creative Commons Attribution Noncommercial License which permits any noncommercial use, distribution, and reproduction in any medium, provided the original author(s) and source are credited.

\section{References}

1. Andersson U, Tracey K (2011) HMGB1 is a therapeutic target for sterile inflammation and infection. Annu Rev Immunol 23:139-162

2. Yao D, Brownlee M (2010) Hyperglycemia-induced reactive oxygen species increase expression of the receptor for advanced glycation end products (RAGE) and RAGE ligands. Diabetes 59:249-255
3. Biscetti F, Straface G, De Cristofaro R et al (2010) High-mobility group box-1 protein promotes angiogenesis after peripheral ischemia in diabetic mice through a VEGF-dependent mechanism. Diabetes 59:1496-1505

4. Straino S, Di Carlo A, Mangoni A et al (2008) High-mobility group box 1 protein in human and murine skin: involvement in wound healing. J Invest Dermatol 128:1545-1553

5. Marchetti C, Di Carlo A, Facchiano F et al (2011) High mobility group box 1 is a novel substrate of dipeptidyl peptidase-IV. Diabetologia. doi:10.1007/s00125-011-2213-6

6. Kirby M, Yu D, O'Connor S, Gorrell M (2010) Inhibitor selectivity in the clinical application of dipeptidyl peptidase-4 inhibition. Clin Sci 118:31-41

7. Dasu MR, Devaraj S, Park S, Jialal I (2010) Increased Toll-like receptor (TLR) activation and TLR ligands in recently diagnosed type 2 diabetic subjects. Diabetes Care 33:861-868

8. Bento C, Pereira P (2011) Regulation of hypoxia-inducible factor 1 and the loss of the cellular response to hypoxia in diabetes. Diabetologia 54:1946-1956

9. Porto A, Palumbo R, Pieroni M et al (2006) Smooth muscle cells in human atherosclerotic plaques secrete and proliferate in response to high mobility group box 1 protein. FASEB J 20:2565-2566

10. Yan XX, Lu L, Peng WH et al (2009) Increased serum HMGB1 level is associated with coronary artery disease in nondiabetic and type 2 diabetic patients. Atherosclerosis 205:544-548

11. Kanellakis P, Agrotis A, Kyaw TS et al (2010) High-mobility group box protein 1 neutralization reduces development of dietinduced atherosclerosis in apolipoprotein E-deficient mice. Arterioscler Thromb Vasc Biol 31:313-319

12. Pachydaki SI, Tari SR, Lee SE et al (2006) Upregulation of RAGE and its ligands in proliferative retinal disease. Exp Eye Res 82:807-815

13. Huisamen B, Genis A, Marais E, Lochner A (2011) Pre-treatment with a DPP-4 inhibitor is infarct sparing in hearts from obese, prediabetic rats. Cardiovasc Drugs Ther 25:13-20

14. Ye Y, Keyes KT, Zhang C, Perez-Polo JR, Lin Y, Birnbaum Y (2010) The myocardial infarct size-limiting effect of sitagliptin is PKA-dependent, whereas the protective effect of pioglitazone is partially dependent on PKA. Am J Physiol Heart Circ Physiol 298:H1454-H1465

15. Catrina S, Okamoto K, Pereira T, Brismar K, Poellinger L (2004) Hyperglycemia regulates hypoxia-inducible factor-1alpha protein stability and function. Diabetes 53:3226-3232

16. Gallagher KA, Liu ZJ, Xiao M et al (2007) Diabetic impairments in NO-mediated endothelial progenitor cell mobilization and homing are reversed by hyperoxia and SDF-1. J Clin Invest 117:1249-1259

17. Fadini GP, Boscaro E, Albiero M et al (2010) The oral dipeptidyl peptidase-4 inhibitor sitagliptin increases circulating endothelial progenitor cells in patients with type 2 diabetes. Diabetes Care 33:1607-1609

18. Ceradini DJ, Yao D, Grogan RH et al (2008) Decreasing intracellular superoxide corrects defective ischemia-induced new vessel formation in diabetic mice. J Biol Chem 283:10930-10938

19. Loot M, Kenter S, Au F, van Galen W, Middelkoop E, Bos J, Mekkes J (2002) Fibroblasts derived from chronic diabetic ulcers differ in their response to stimulation with EGF, IGF-I, $\mathrm{bFGF}$ and PDGF-AB compared to controls. Eur J Cell Biol Mar 81:153-160

20. Tchaikovski V, Olieslagers S, Bohmer FD, Waltenberger J (2009) Diabetes mellitus activates signal transduction pathways resulting in vascular endothelial growth factor resistance of human monocytes. Circulation 120:150-159

21. Thangarajah H, Yao D, Chang EI et al (2009) The molecular basis for impaired hypoxia-induced VEGF expression in diabetic tissues. Proc Natl Acad Sci U S A 106:13505-13510 